\title{
Runx2 Regulates the Gene Network Associated with Insulin Signaling and Energy Homeostasis
}

\author{
Mitra Adhami $^{\mathrm{a}}$ Farah Y. Ghori-Javed $^{\mathrm{a}}$ Haiyan Chen ${ }^{\mathrm{a}}$ Soraya E. Gutierrez ${ }^{\mathrm{b}}$ \\ Amjad Javed $^{\text {a }}$ \\ aDepartment of Oral and Maxillofacial Surgery, School of Dentistry, University of Alabama at Birmingham, \\ Birmingham, Ala., USA; 'b Departamento de Biología Molecular, Universidad de Concepción, Concepción, Chile
}

\section{Key Words}

Runx2 - Insulin signaling • Adipocyte differentiation •

Energy metabolism $\cdot$ Gene microarray

\begin{abstract}
Glucose intolerance seen in metabolic disorders, such as type II diabetes, is commonly associated with improper execution of the insulin signaling pathway, as well as an imbalance of bone and fat tissues, such that a gain in adipose tissue occurs at the expense of bone loss. Fat-producing adipocytes and bone-forming osteoblasts stem from a common mesenchymal progenitor cell. Runx 2 positively regulates the commitment of the mesenchymal cell toward osteogenesis, but its effects on energy homeostasis and the insulin signaling pathway are unknown. To investigate the connection, focused microarray profiling of genes associated with the insulin signaling pathway was performed on calvarial cells from Runx2-null embryonic mice and 3T3-L1 preadipocytes treated with control and insulin-containing media. The microarray showed that addition of insulin resulted in a robust induction of genes (>95\%) in 3T3-L1 cells. Surprisingly, Runx2-null cells cultured in control media were at an elevated state of energy metabolism and addition of insulin resulted in a marked suppression of genes required for insulin
\end{abstract}

signaling. Clustering analysis revealed that the suppression occurred at all stages of the insulin pathway, from the receptors and transducers to nuclear effectors and target genes. Taken together, these results demonstrate that Runx 2 is central for transduction and execution of the insulin regulatory signal. In conclusion, Runx 2 actively regulates the gene network required for glucose metabolism and energy homeostasis in mesenchymal cells.

Copyright $\odot 2011$ S. Karger AG, Basel

\section{Abbreviations used in this paper}

cAMP cyclic adenosine monophosphate

CAP1 cyclase-associated protein 1

$\mathrm{C} / \mathrm{EBP} \alpha \quad \mathrm{CAAT}$ enhancer binding protein alpha

$\mathrm{C} / \mathrm{EBP} \quad \mathrm{CAAT}$ enhancer binding protein beta

Glut4 glucose transporter 4

IGF1R insulin-like growth factor 1 receptor

IR insulin receptor

IRS insulin receptor substrate

LDLR low-density lipoprotein receptor

MAPK mitogen-activated protein kinase

PI3K phosphoinositide 3-kinase

PPAR $\gamma \quad$ peroxisome proliferator-activated receptor gamma

Srebf1 sterol regulatory element binding factor 1

\section{KARGER}

() 2011 S. Karger AG, Basel

Fax +4161306 1234

E-Mail karger@karger.ch

www.karger.com
Accessible online at: www.karger.com/cto
Dr. Amjad Javed

Department of Oral and Maxillofacial Surgery

School of Dentistry, University of Alabama at Birmingham

SDB 714, 1530 3rd Ave. South, Birmingham, AL 35294-0007 (USA)

Tel. +1 205996 5124, E-Mail javeda@ uab.edu 


\section{Introduction}

During aging, obesity and metabolic disorders such as type II diabetes, an increased adiposity with a concomitant decrease in bone is commonly noted [Duque, 2008; Isidro and Ruano, 2010]. Bone and fat are tissue types that stem from the mesenchymal progenitor cell. In fact, bone marrow mesenchymal cells have the potential to give rise to both osteoblasts and adipocytes [de Paula et al., 2010]. Commitment toward these tissue lineages from the common progenitor cell is controlled by the transcription factors CAAT enhancer binding protein beta $(\mathrm{C} / \mathrm{EBP} \beta)$, CAAT enhancer binding protein alpha $(\mathrm{C} / \mathrm{EBP} \alpha)$, and peroxisome proliferator-activated receptor gamma (PPAR $\gamma$ ) for adipogenesis [Cao et al., 1991; Kim et al., 2010] and Runx2 and Osterix for osteogenesis [Komori et al., 1997; Nakashima et al., 2002; Lian et al., 2004].

Metabolic disorders, aging, and obesity often have an underlying theme of improper insulin signaling which controls glucose homeostasis and energy metabolism [Cusi et al., 2000]. Aged and obese individuals and patients with metabolic disorders show insulin resistance and an inability to clear glucose from the bloodstream. The insulin pathway is involved in balancing the production of glucose from the liver and utilization of this glucose by all organs. Insulin produced by pancreatic $\beta$ cells is recognized by all target organs through the insulin receptor (IR) and insulin-like growth factor receptor 1 (IGF1R). In fact, although the levels vary, almost all mammalian tissues express the IR [Kahn et al., 1988]. The liver and adipose tissue contain the highest number of IR $(>300,000)$ and are considered 2 of the major insulin-dependent tissues.

In healthy individuals, $\beta$ cells from the pancreas secrete insulin in response to elevated glucose levels, which then signal peripheral tissues to uptake circulating glucose into their cells [Cheatham and Kahn, 1995]. Metabolic disorders are acquired through insufficient production or recognition of insulin by target tissues [Moller and Flier, 1991]. A key set of intracellular transducers is a group of proteins termed the IR substrates (IRS). In a properly functioning pathway, the IR is expected to rapidly phosphorylate IRS1 and Shc at tyrosine residues to stimulate the activation of the Ras pathway. This stimulation is also aided by IRS2. These substrates comprise the first step of the insulin signaling cascade and are used in both mitogen-activated protein kinase (MAPK) and phosphoinositide 3-kinase (PI3K) pathways [Yang et al., 2010].

The MEK/ERK pathway consists of a cascade of serine/ threonine protein kinases, and it is utilized in regulation of the cell cycle through the control of transcription factors. Defects in the MEK/ERK pathway often lead to the development of cancer. The PI3K pathway allows for lipid storage in the form of glycogen through transfer of the glucose transporter 4 (Glut4) to the plasma membrane for glucose import [Cong et al., 1997; Baumann et al., 2000]. The final step in the execution of the insulin signal is the regulation of target genes by nuclear effectors. The major nuclear transcription factors include members of the CAAT enhancer binding protein family (C/EBPs) and PPAR $\gamma$. Although insulin is known for its primary effects on glucose homeostasis, correct reception and signaling in the insulin pathway are required for many biological functions, such as the normal storage of fuels and cellular growth and differentiation [Czech, 1977].

Bone is becoming more recognized as a key insulinutilizing tissue and an important player in glucose homeostasis [Cheatham and Kahn, 1995]. Bone formation and remodeling is a dynamic process that requires tremendous energy expenditure. Acknowledging that bone cells are major stakeholders in insulin signaling, we used Runx2, a molecule obligatory for bone tissue, as a paradigm to investigate its role in energy metabolism.

\section{Materials and Methods}

\section{Cell Culture}

Runx2-null cells from the calvaria of E17.5 Runx2 global knockout mice [Komori et al., 1997] and 3T3-L1 preadipocytes were cultured in growth media $(\alpha \mathrm{MEM}, 10 \% \mathrm{FBS}, 1 \%$ penicillin streptomycin, and $1 \%$ L-glutamine). Upon confluence, cells were fed every other day with either control media or control media supplemented with $880 \mathrm{nmol}$ of insulin for a total of 10 days.

\section{Total RNA Isolation}

Total RNA was isolated from cells according to the SABiosciences Array Grade Total RNA Isolation Kit protocol. All cells from each $10-\mathrm{cm}$ plate were harvested and pelleted by centrifugation. Cells were then lysed, cleaned, and desalted. RNase-free water was used to elute total RNA from each sample. UV spectrophotometry was used to quantify and determine the purity of each sample. Samples required a 260/280 ratio of 2.0 and a 260/230 ratio of 1.7 for adequate purity.

\section{cRNA Target Labeling for Oligo GEArray Hybridization}

Total RNA was first converted to cRNA according to the SABiosciences TrueLabeling-AMP 2.0 manual. RNA was added to an annealing mixture and then to a cDNA synthesis master mix, gently mixed, and allowed to incubuate. cRNA was synthesized by adding and incubating with an RNA polymerase master mix to the cDNA. cRNA was then purified and quantification and quality were assessed using UV spectrophotometry. A minimum of $4 \mu \mathrm{g}$ of labeled cRNA for each sample was used for probing. 
Fig. 1. Differential response to insulin by Runx2-deficient mesenchymal cells. Both 3T3-L1 and Runx2-null cells were treated in the presence and absence of $880 \mathrm{nmol}$ insulin for 10 days. Total RNA was isolated and blotted for focused microarray of the 128 genes known to be involved in the insulin signaling pathway. The expression level of each gene was determined by a 4-probe set. Densitometric analysis of signal intensity was performed with an Alpha Innotech imaging system and used to generate a heat map. Two independent membranes for each cell type were probed and representative images of the histograms taken simultaneously are shown.

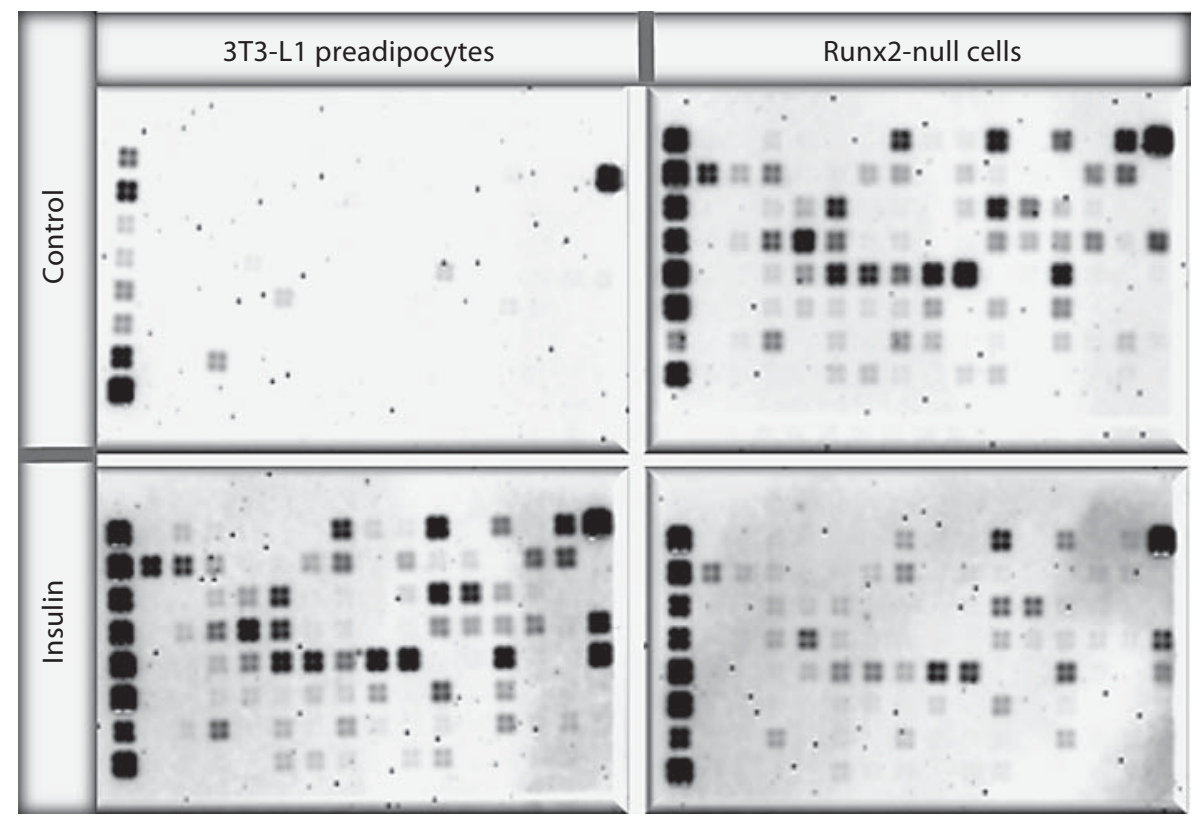

Oligo GEArray Microarray

cRNA was hybridized to the mouse insulin signaling pathway oligo GEArray DNA microarray containing 128 genes according to the SABiosciences HybTube standard protocol. cRNA was allowed to hybridize to the membrane overnight and washed; chemiluminescence was used to detect the signal. Images of blots were captured simultaneously using an Alpha Innotech system.

\section{Results and Discussion}

Runx 2 is essential for the development and biological functions of osteoblasts. The synthesis of mineralized matrix by osteoblasts throughout our lifetime demands tremendous energy expenditure. To better understand the effects of Runx 2 on glucose metabolism and energy homeostasis, we utilized a Runx2-null cell model. Calvarium-derived mesenchymal cells from Runx2-null mice were treated with insulin. Transduction of the insulin signal is complex and involves multiple interactions of regulatory cues along the entire pathway. Thus, we used a focused gene microarray that consists of 128 genes representing all key steps and molecules involved in the execution of the insulin signal (fig. 1). Runx2-null cells, though isolated from calvarial tissue, preferentially commit toward the adipocyte lineage but not toward osteoblasts. In contrast, calvarial cells isolated from wild-type littermates fail to commit to the adipocyte lineage and differentiate toward osteoblasts. The effects of insulin on 3T3-L1 preadipocytes and how they assimilate insulin's anabolic actions are well understood. Furthermore, 3T3L1 cells which are derived from wild-type mice are developmentally close to Runx2-null cells in that they were isolated from E14 mice. Therefore, for comparison of insulin signaling we utilized 3T3-L1cells as a control.

Total RNA was isolated in parallel from both cell types and hybridized simultaneously with the membrane containing the 128 imprinted genes. Each gene was represented with 4 independent probe sets to eliminate any potential nonspecific signal (fig. 1). Since all experimental procedures for Runx2-null and 3T3-L1 cells were performed together, the comparative changes could be quantified with maximum confidence. Our analysis shows that Runx2-null control cells are at an astonishingly high state of basal metabolism, consistently matching (or surpassing) expression level of genes induced by insulin in preadipocytes. Interestingly, a closer look at gene expression revealed that the two paths of insulin signaling, though both robustly induced in Runx2-null control cells, were differentially altered by treatment of insulin. Genes comprising the MAPK pathway and associated with cell proliferation and survival (i.e. Grb and Sos) were either unchanged or downregulated to match the expression level of insulin-treated preadipocytes. In sharp contrast, genes comprising the PI3K pathway, and associated with glucose import and adipogenesis (i.e. Pik3r3 and GSK3), were downregulated by insulin treatment in Runx2-null cells, such that rather than maintaining the level comparable to insulin-treated preadipocytes, gene 


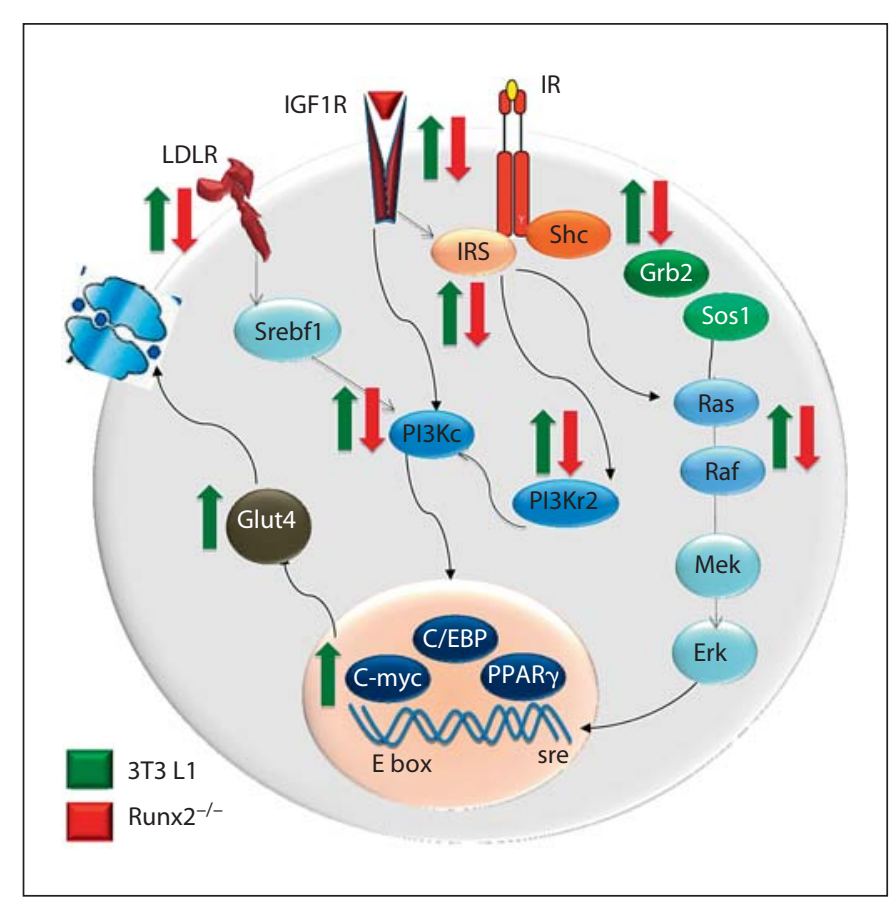

Fig. 2. Runx2-deficient cells exhibit suppression of genes by insulin at multiple stages of the signaling pathway. Proteins at different stages of the pathway from the receptor, transducer, nuclear effecter, and target gene are shown. Expressions of respective genes are downregulated upon addition of insulin to Runx2-null cells (indicated by red arrows), while insulin robustly induces these genes in 3T3-L1 cells (indicated by green arrows).

Table 1. Comparison of insulin pathway gene levels in control 3T3-L1 and Runx2-null cells

Fold changes in baseline gene expression in Runx2-null cells (Runx2/3T3-L1)

\begin{tabular}{|c|c|c|c|c|c|c|c|}
\hline \multicolumn{2}{|c|}{ receptors } & \multicolumn{2}{|c|}{ transducers } & \multicolumn{2}{|c|}{$\begin{array}{l}\text { transcription } \\
\text { factors }\end{array}$} & \multicolumn{2}{|l|}{ target genes } \\
\hline IR & 2 & IRS1 & 6 & $\mathrm{C} / \mathrm{EBP} \alpha$ & 3 & Glut4 & 7 \\
\hline IGF1R & 16 & IRS2 & 4 & Srebf1 & 6 & Adiponectin & 6 \\
\hline LDLR & 6.5 & Shc1 & 5 & $\operatorname{PPAR} \gamma$ & 2.5 & Leptin & 10 \\
\hline
\end{tabular}

expression was actively suppressed, and approached expression levels quantified in control preadipocytes.

\section{Insulin Robustly Induces Genes Associated with}

Insulin Signaling in Preadipocytes

The mouse 3T3-Ll cell line rapidly differentiates to an adipocyte phenotype when postconfluent cells are treated with adipogenic media and sustained on insulin-supplemented media for more than a week. Our analysis revealed no expression or barely detectable levels of insulin pathway genes in control cells. However, insulin-treated 3T3-L1 cells showed robust upregulation (2- to 18-fold) in greater than $95 \%$ of genes in the insulin pathway (fig. 1). Addition of insulin induced the expression of both IR and IGF1R by 2 - and 13 -fold, respectively (table 1 ). Consistent with its anabolic role, insulin treatment also resulted in upregulation of the low-density lipoprotein receptor (LDLR) by 3.5-fold compared to control cells.

The intracellular transducers in the insulin signal, i.e. the IRS, were all induced upon addition of insulin to 3T3L1 cells. For instance, IRS1 and Shc1 were induced 7- and 5 -fold, respectively. Members of the Ras family, a pathway involved in the regulation of proliferation and cell differentiation [Yonezawa et al., 1994], were also upregulated 7-fold with insulin treatment (table 1).

Key transcription factors responsible for integration of the anabolic action of insulin were all induced in 3T3-L1 insulin-treated cells. PPAR $\gamma$ was induced 2 -fold, Jun was induced 3-fold, and sterol regulatory element binding factor 1 (Srebf1) was induced the maximum 5-fold. Downstream target genes were also induced with insulin treatment so that adiponectin, Glut4, and leptin were induced 6-, 7-, and 10-fold, respectively (table 1). Upregulation of the gene network in 3T3-L1 cells in response to insulin mimics the anabolic response in a healthy individual during energy metabolism.

\section{Runx2 Deficiency Is Associated with Increased \\ Expression of the Insulin Gene Network}

Surprisingly, our analysis revealed significantly elevated baseline expression levels of all genes in Runx2null control cells when compared to 3T3-L1 control cells. These levels in control Runx2-null cells were almost identical to those seen in the insulin-stimulated 3T3-L1 cells. While both cell types express the IR and IGF1R in basal condition, Runx2-null cells express these receptors 2and 13-fold more compared to 3T3-L1 cells (fig. 1; table 2). Additionally, the LDLR was noted to be expressed in Runx2-null cells at least 6.5-fold more when compared to 3T3-L1 cells.

The baseline expression of postreceptor transducers was also at very high levels in Runx2-null cells, particularly IRS1, IRS2, and Shc1. These substrates comprise the first step of the insulin signaling cascade and were induced 4- to 6-fold in Runx2-null cells compared with 3T3-L1 control cells (table 2). 
Table 2. Insulin enhances expression of insulin signaling in 3T3L1 cells

Fold changes in gene expression by insulin treatment in 3T3-L1 cells (insulin/control)

\begin{tabular}{|c|c|c|c|c|c|}
\hline receptors & transducers & \multicolumn{2}{|c|}{$\begin{array}{l}\text { transcription } \\
\text { factors }\end{array}$} & \multicolumn{2}{|l|}{ target genes } \\
\hline 2 & IRS1 & Jun & 3 & Glut4 & 7 \\
\hline IGF1R 13 & Rras2 & Srebf1 & 5 & Adiponectin & 6 \\
\hline LDLR & Shc1 & $\operatorname{PPAR} \gamma$ & 2 & Leptin & 10 \\
\hline
\end{tabular}

The baseline gene induction was also evident in the transcription factors $\mathrm{C} / \mathrm{EBP} \alpha$ and PPAR $\gamma$, which were upregulated 3- and 2.5-fold, respectively. Interestingly, although all transcription factors in the microarray were induced up to 3.5-fold, in Runx2-null cells the maximum elevation (6-fold) was noted for Srebf1. Srebf1 is known to flank the LDLR as well as other genes involved in sterol biosynthesis [Walker et al., 2010]. Thus, increased expression of LDLR and Srebf1 in Runx2-null cells suggests an increased rate of lipid and cholesterol metabolism. Consistent with the enhanced expression of transcription factors, downstream target genes in the insulin signaling pathway such as Glut4, and the key adipokines adiponectin and leptin were all induced 6- to 10-fold in Runx2-null cells when compared to 3T3-L1 preadipocytes (table 2).

As noted earlier, wild-type cells isolated from the same litter would not be analogous to Runx2-null cells as they exhibit a different developmental status. Calvarial cells from wild-type littermates are progressively mature osteoblasts, while Runx2-null cells represent only primitive mesenchymal cell features and preferentially commit toward the adipocyte lineage. Furthermore, wild-type cells do not maintain the capacity to undergo adipogenesis and thus would not be a comparable control against preadipocytic Runx2-null cells. In summary, expression levels of genes in control Runx2-null cells nearly match those of insulin-treated 3T3-L1 preadipocytes. This induction of genes in the insulin pathways suggests that an increased basal state of energy metabolism is associated with Runx2 deficiency.

\section{Insulin Suppresses Genes Associated with the Insulin}

Signaling Pathway in Runx2-Null Cells

With the increased metabolic state of Runx2-null mesenchymal cells, we anticipated a robust activation of the insulin pathway upon addition of insulin. To our sur-
Table 3. Changes in expression of insulin signaling genes in insulin-treated Runx2-null cells

Relative changes in insulin-exposed Runx2-null cells (insulin/control)

\begin{tabular}{|c|c|c|c|c|c|}
\hline \multicolumn{2}{|c|}{ receptors } & \multirow{2}{*}{$\begin{array}{l}\text { transducers } \\
\text { IRS1 } 30 \%\end{array}$} & $\begin{array}{l}\text { transcription } \\
\text { factors }\end{array}$ & \multicolumn{2}{|l|}{ target genes } \\
\hline IR & $20 \%$ & & $\mathrm{C} / \mathrm{EBP} \alpha \quad \mathrm{NC}$ & Glut4 & $\mathrm{NC}$ \\
\hline IGF1R & $50 \%$ & CAP1 30\% & Srebf1 & Adiponectin & $\mathrm{NC}$ \\
\hline LDLR & $20 \%$ & Rras $250 \%$ & PPAR $\gamma$ & Leptin & $60 \%$ \\
\hline
\end{tabular}

prise, insulin-treated Runx2-null cells showed downregulation of genes throughout the pathway (fig. 1). Shockingly, addition of insulin to Runx2-null cells suppressed the expression of the IR and IGF1R (table 3). In fact, all receptors in the microarray were downregulated, with the exception of Adrald which showed no significant change.

Insulin treatment of the Runx2-null cells also caused significant downregulation of intracellular transducers. We observed a $30-40 \%$ decrease in the expression of IRS1 and Shc; however, IRS2 showed no significant decrease. Transducers associated with members of the Ras cascade (Rras2) were also downregulated up to $50 \%$. Our results are consistent with earlier reports that IRs with an impaired ability to appropriately phosphorylate IRS1 and Shc are also unable to fully activate the Ras pathway [Tadahiro et al., 1994]. The downregulation in the insulin substrates could be associated with this decrease in the expression of the Ras pathway. Cyclase-associated protein 1 (CAP1) is associated with the cyclic adenosine monophosphate (cAMP) pathway, and it is utilized for recruiting Glut4 to the plasma membrane as an alternative to the PI3K pathway [Baumann et al., 2000]. Interestingly, CAP1 was considerably downregulated $(50 \%)$ in insulin-treated Runx2-null cells, yet it was substantially induced (16-fold) in insulin-treated 3T3-L1 cells. It is important to note that control Runx2-null cells exhibited an 18-fold elevated baseline expression of CAP1 when compared to 3T3-L1 control cells.

Most transcription factors in insulin-treated Runx2null cells showed no significant change in their level and continued to maintain an elevated state of expression like the Runx2-null control cells (table 3). However, Srebf1 was strikingly downregulated by $70 \%$. Target genes in the pathway also showed no change with insulin treatment, with the exception of leptin which was decreased by $60 \%$ 
in Runx2-null cells. These results demonstrate that insulin-mediated suppression in Runx2-deficient cells primarily targets receptors and transducers (fig. 2).

Taken together, our results provide evidence that control of the mesenchymal cell lineage commitment by Runx 2 entails regulation of the gene circuitry associated with energy homeostasis.

\section{Acknowledgment}

This work was supported by National Institute of Health grant RO1 AG030228.

\section{References}

Baumann, C., V. Ribon, M. Kanzaki, D. Thur- De Paula, F., M. Horowitz, C. Rosen (2010) Novel mond, S. Mora, S. Shigematsu, P. Bickel, J. Pessin, A. Saltiel (2000) CAP defines a second signalling pathway required for insulinstimulated glucose transport. Nature 407: 202-207.

Cao, Z., R.M. Umek, S.L. McKnight (1991) Regulated expression of three C/EBP isoforms during adipose conversion of 3T3-L1 cells. Genes Dev 5: 1538-1552.

Cheatham, B., C. Kahn (1995) Insulin action and the insulin signaling network. Endocr Rev 16: 117-142.

Cong, L., H. Chen, Y. Li, L. Zhou, M. McGibbon, S. Taylor, M. Quon (1997) Physiological role of Akt in insulin-stimulated translocation of GLUT4 in transfected rat adipose cells. Mol Endocrinol 11: 1881-1890.

Cusi, K., M. Katsumi, O. Abdullah, M. Pendergrass, M. Elizabeth, T. Pratipanawatr, R. DeFronzo, C. Kahn. L. Mandarino (2000) Insulin resistance differentially affects the PI 3-kinase- and MAP kinase-mediated signaling in human muscle. J Clinical Invest 105: 311-320.

Czech, M. (1977) Molecular basis of insulin action. Annu Rev Biochem 46: 359-384. insights into the relationship between diabetes and osteoporosis. Diabetes Metab Res Rev 26: 622-630.

Duque, G. (2008) Bone and fat connection in aging bone. Curr Opin Rheumatol 20: 429434.

Isidro, M., B. Ruano (2010) Bone disease in diabetes. Curr Diabetes Rev 6: 144-155.

Kahn, C., M. White (1988) The insulin receptor and the molecular mechanisms of insulin actions. J Clinical Invest 82: 1151-1156.

Kim, S., J. Ha, S. Yun, E. Kim, S. Chung, K. Hong, C. Kim, S. Bae (2010) Transcriptional activation of peroxisome proliferator-activated re ceptor-gamma requires activation of both protein kinase A and Akt during adipocyte differentiation. Biochem Biophys Res Commun 399: 55-59.

Komori, T., H. Yagi, S. Normura, et al. (1997) Targeted disruption of Cbfa1 results in a complete lack of bone formation owing to maturational arrest of osteoblasts. Cell 89: $755-764$.

Lian, J., A. Javed, S. Zaidi, C. Lengner, M. Montecino, A. van Mijnen, J. Stein, G. Stein (2004) Regulatory controls for osteoblast growth and differentiation: role of Runx/ Cbfa/AML factors. Crit Rev Eurkaryot Gene Expr 14: 1-41.
Moller, D., J. Flier (1991) Insulin resistance: mechanisms, syndromes, and implications. N Engl J Med 325: 938-948.

Nakashima, K., X. Zhou, G. Kunkel, Z. Zhang, J.M. Deng, R.R. Behringer, B. de Crombrugghe (2002) The novel zinc finger-containing transcription factor osterix is required for osteoblast differentiation and bone formation. Cell 108: 17-29.

Tadahiro, K., S. Kenta, S. Hara, M. Nakafukun, S. Okabayashi, T. Kadowaki, Y. Kaziron, M. Kasuga (1994) Signal transduction pathways from insulin receptors to Ras. J Biol Chem 269: 4634-4640.

Walker, A., F. Yang, K. Jiang, et al. (2010) Conserved role of SIRT1 orthologs in fasting-dependent inhibition of the lipid/cholesterol regulator SREBP. Genes Dev 24: 1403-1417.

Yang, J., X. Zhang, W. Wang, J. Liu (2010) Insulin stimulates osteoblast proliferation and differentiation through ERK and PI3K in MG63 cells. Cell Biochem Funct 28: 334-341.

Yonezawa, K., A. Ando, Y. Kaburagi, R. Yamamoto-Honda, T. Kitamura, K. Hara, M. Nakafuku, Y. Okabayashi, T. Kadowaki, Y. Kaziro (1994) Signal transduction pathways from insulin receptors to Ras: analysis by mutant insulin receptors. J Biolo Chem 269: 4634-4640. 